\title{
Anabases
}

ANABASES Traditions et réceptions de l'Antiquité

$8 \mid 2008$

Varia

Échos contemporains de la Guerre de Troie : l'Iliade dans la bande dessinée, le cinéma, la sciencefiction, la littérature de jeunesse (1956-2007)

\section{Audy Rodriguez}

\section{(2) OpenEdition}

Journals

Édition électronique

URL : http://journals.openedition.org/anabases/164

DOI : 10.4000/anabases.164

ISSN : 2256-9421

Éditeur

E.R.A.S.M.E.

Édition imprimée

Date de publication : 1 octobre 2008

Pagination : 137-150

ISSN : $1774-4296$

\section{Référence électronique}

Audy Rodriguez, «Échos contemporains de la Guerre de Troie : I'lliade dans la bande dessinée, le cinéma, la science-fiction, la littérature de jeunesse (1956-2007) », Anabases [En ligne], 8| 2008, mis en ligne le 01 juillet 2011, consulté le 20 octobre 2019. URL : http://journals.openedition.org/anabases/ 164 ; DOI : 10.4000/anabases. 164 
Anabases 8 (2008), p. 137-150.

\author{
Échos contemporains \\ de la Guerre de Troie : l'Iliade \\ dans la bande dessinée, le cinéma, \\ la science-fiction, la littérature \\ de jeunesse (1956-2007)
}

AUDY RODRIGUEZ

Le ThÈME DE LA REPRÉSENTATION DE LA GueRre DE Troie dans les productions culturelles contemporaines pose la question des motivations, notamment idéologiques, et des modalités formelles qui ont influé sur le contenu de ces œuvres. S’il est évident que les auteurs contemporains qui ont réalisé celles-ci ont été inspirés, voire conditionnés, à des degrés divers par l'Iliade et la tradition qui en est issue au fil des siècles, on peut toujours s'interroger sur l'intérêt qu'ils avaient à mobiliser une référence culturelle, aussi renommée soit-elle, vieille de presque trois mille ans et, qui plus est, largement revisitée depuis. Il ne peut s'agir là d'une adaptation mécanique à des supports nouveaux comme le cinéma ou la bande dessinée. Le fait est, plus sûrement, que, quel que soit le support envisagé, l'Iliade nous parle toujours et que c'est cette capacité qui incite les auteurs contemporains à s'y intéresser, recourant dans leur tentative de valorisation de ce potentiel à divers procédés et démarches. C'est à ces dernières que nous nous intéresserons d'abord : à travers la présentation de quatre catégories - la retranscription, l'adaptation, la reformulation, et la transposition -, nous verrons comment une œuvre éminemment classique a, à travers des réalisations diverses, épousé les évolutions protéiformes de la production culturelle contemporaine. Ensuite, afin de mieux saisir la spécificité des apports contemporains, l'analyse sera poursuivie à travers trois axes principaux illustrant chacun une notion qui nous semble être constitutive du contexte des mentalités contemporaines : la réflexivité, la rétrospectivité et le désenchantement. Enfin, nous tenterons d'illustrer l'interactivité, au sein des œuvres, entre ces diverses notions.

En préambule, toutefois, nous présenterons succinctement notre corpus d'étude. D’abord, nous avons retenu quatre bandes dessinées : Ilios, de Hosegawa Hironi, éditée notamment sur Internet, qui date de 1997 pour le début de sa parution ; Le dernier Troyen de Valérie Mangin et Thierry Démarez ainsi que L'áge de bronze de Eric 
Shanower, deux bandes dessinées en plusieurs tomes dont les premiers sont parus en 2004 ; La colère d'Achille de Camille Le Gendre (2006). S'y ajoutent, le roman en deux tomes intitulés Ilium et Olympos de Dan Simmons (2004 et 2005). On y ajoutera encore l'Iliade traduite et adaptée du grec ancien par Michel Woronoff et illustrée par Bruno Pilorget (2007). Pour ce qui est des films, nous nous en tiendrons aux réalisations suivantes : Hélène de Troie de Robert Wise (1956), La guerre de Troie de Giorgio Ferroni (1960), La colère d'Achille de Mario Girolami (1962), Hélène de Troie de John Kent Harrison (2003), et enfin Troy de Wolfgang Petersen (2004). Cette liste situe la totalité des œuvres étudiées dans le contexte chronologique des cinquante dernières années.

\section{Retranscription, adaptation, reformulation, transposition : des frontières friables}

L'Iliade "ne relate que " la colère d'Achille : le récit débute à la neuvième année du conflit avec l'épisode de la peste lancée par Apollon sur les Achéens, ce qui indirectement sera à l'origine de la dispute entre Agamemnon et Achille et donc de la susdite colère de ce dernier ; il se termine par la remise du corps d'Hector à Priam par Achille. Tous les autres épisodes de la Guerre de Troie que nous connaissons, qu'ils se situent en amont ou en aval du moment illustré par l'Iliade, sont empruntés à d'autres sources ${ }^{1}$. Il est alors possible pour les auteurs de se référer à plusieurs traditions datant de différentes époques pour aborder ce sujet, une observation qui vaut tant pour des sources datant de l'Antiquité que pour d'autres beaucoup plus récentes.

De fait, le corpus des œuvres étudiées traduit une fluctuation des bornes temporelles délimitant la Guerre de Troie en amont, avec la présence d'éléments remontant aux origines plus ou moins lointaines de la Guerre ${ }^{2}$, mais aussi de façon très prolongée en aval. Cette dernière tendance se manifeste notamment dans des œuvres qui présentent le déroulement de la Guerre dans un cadre futuriste ou qui intègrent des éléments rapportés par des sources postérieures à la tradition grecque proprement dite ${ }^{3}$.

Pour ce qui est de notre corpus, La colère d'Achille de Mario Girolami débute au cours de la neuvième année de siège avec la capture de Chryséis, fille de Chrysès, par

1 A. EISSEN, Les mythes grecs, éd Belin, 1993. Plusieurs poètes avaient traité chacun un moment de la guerre de Troie. Dans l'ordre des événements racontés, énumérons les Chants Cypriens (les origines et le début de la guerre), l'Éthiopide (de la fin de l'Iliade à la dispute autour des armes d'Achille), la petite Iliade (de la mort d'Ajax à l'entrée des Grecs dans Troie), la Prise d'Ilion et les Retours (des différents chefs grecs, à l'exception d'Ulysse puisque l'Odyssée lui était consacrée).

2 C'est le cas par exemple de la pomme de la déesse discorde, la prophétie concernant le destin d'Alexandre-Pâris, du choix de Pâris, de l'enlèvement d'Hélène...

3 C'est le cas du Dernier Troyen, d'Ilium ou encore d'allusions, dans La Guerre de Troie ou Troy, au destin d'Énée après la chute de Troie. 
les Achéens; le film se termine avec la remise par Achille du corps d'Hector à Priam. La bande dessinée du même nom réalisée par Camille Le Gendre débute avec la requête de Chrysès auprès des Achéens au sujet de la libération de sa fille et s'achève sur les funérailles d'Hector. Le livre de Michel Woronoff intitulé l'Iliade s'inscrit dans les mêmes bornes narratives. Le Guerre de Troie de Giorgio Ferroni nous plonge lui aussi au cours de la neuvième année de siège, mais se termine avec la prise de Troie et la fuite du véritable héros du film, Énée. Dans Hélène de Troie de Robert Wise, le récit commence en amont de l'enlèvement d'Hélène par Pâris avec un contexte géopolitique déjà tendu entre Troyens et Achéens dans lequel cependant les négociations diplomatiques restent possibles. Celles-ci seront d'ailleurs à l'origine de la venue de Pâris à Sparte. Le film Troy de Wolfgang Petersen suit une trame similaire : après avoir exposé les menées hégémoniques d'Agamemnon en Grèce propre, le film présente l'ambassade d'Hector et Pâris à Sparte ; cette réalisation s'achève elle aussi avec une prise de Troie dans laquelle les auteurs laissent libre cours à leur liberté narrative. Hélène de Troie, de John Kent Harrison remonte aux origines, avec la naissance de Pâris présagée comme néfaste par sa sœur Cassandre, puis la jeunesse de celui-ci comme bouvier et l'épisode de sa confrontation avec les trois déesses; le film se termine lui aussi par la prise de Troie. De même, Eric Shanower et Hosegawa Hironi ont choisi dans leurs bandes dessinées respectives, L'áge de bronze et Ilios, de narrer des épisodes de la jeunesse de Pâris ; ces deux ouvres étant toujours en cours de parution, il n'est à ce jour pas possible de dire à quel stade de la trame les auteurs termineront leurs récits. Ilium et Olympos, le roman en deux tomes de Dan Simmons, débute par la dispute entre Achille et Agamemnon; cependant, du fait de l'entremêlement des temporalités au sein de l'œuvre, celle-ci finit par s'écarter totalement de la trame traditionnelle de la guerre de Troie (infra). Le dernier Troyen, une bande dessinée qui se décline elle aussi en plusieurs tomes, tient une place particulière puisque les auteurs font débuter leur œuvre au moment où les Troyens s’apprêtent à faire pénétrer dans leur cité le "cheval laissé en don " par des Achéens qui, comme dans la trame originelle, semblent avoir pris la fuite. Outre le fait que la trame se déroule dans un contexte futuriste, l'objectif poursuivi dans cette œuvre, et notamment dans le premier tome intitulé Le cheval de Troie, est d'établir la jonction entre ce que l'on pourrait appeler la légende de la Guerre de Troie, l'Odyssée d'Homère et l'Énéide de Virgile. Il n'est donc pas étonnant que dans cette bande dessinée le protagoniste ne soit pas Achille, ni même Ulysse, mais Énée. De plus, si la fin du siège et la prise de Troie constituent pleinement la trame du premier tome, il faut reconnaitre que les quatre tomes suivants sont plutôt structurés par des emprunts et des références provenant de l'Énéide et de l'Odyssée.

L'observation des titres fournit aussi des indices. Ainsi, des titres comme l'Iliade ou La colère d'Achille (film de Mario Girolami, bande dessinée de Camille Le Gendre, livre de Michel Woronoff) marquent au minimum la volonté de s'inscrire dans une certaine filiation par rapport à la tradition. À l'inverse, dans Ilium et Olympos de Dan Simmons, L'âge de bronze de Eric Shanower, Le dernier Troyen de Valérie Mangin et 
Thierry Démarez ou encore, mais dans une moindre mesure, le manga sur support papier Ilios de Hosegawa Hironi, l'originalité des titres fait écho à des libertés plus grandes prises par rapport à la trame traditionnelle de l'Iliade. Dans le même ordre d'idée, les œuvres sur la Guerre de Troie où le personnage principal n'est pas Achille sont marquées par une prise de distance par rapport à l'œuvre d'Homère : ainsi, les deux films intitulés Hélène de Troie.

Le recoupement de ces observations avec d'autres permet d'éclairer la démarche suivie par les auteurs et conduit à une répartition de ces productions en quatre groupes.

Le premier, celui des retranscriptions, est constitué par des œuvres dont les auteurs ont cherché à reproduire le plus fidèlement possible, sur le support choisi, la matière qui leur a servi de source. Ainsi, dans La colère d'Achille de Mario Girolami, la volonté de fidélité s'accompagne d'une démarche didactique que traduit, au début, la présence d'une voix-off qui explique le contexte sur fond d'une carte de l'Égée. En outre, le film, qui suit la trame de la colère d'Achille, est parsemé de citations ou extraits de l'Iliade.

La bande dessinée de Camille Le Gendre intitulée La colère d'Achille semble elle aussi entrer dans cette catégorie. Dès la couverture, outre le titre, l'inscription " adapté de l'Iliade d'Homère " le suggère; à l'intérieur de celle-ci, on relève également la présence d'extraits de l'œuvre originale traduits en français et composés de caractères blancs, le tout sur un fond noir de forme rectangulaire. La spécificité de cette œuvre réside cependant dans le graphisme, marqué par des traits qui rappellent la peinture. En effet, cette œuvre est aux antipodes du graphisme de l'école franco-belge du type Hergé ou Jacques Martin, elle-même largement influencée par le classicisme issu de la tradition de l'art grec. En ce qui concerne l'œuvre de Le Gendre, on pourrait dire qu’à supposer que l'auteur ait puisé au sein de cet héritage artistique antique, son influence serait celle des statues de type Kouroi des $\mathrm{VII}^{\mathrm{e}}$ et $\mathrm{VI}^{\mathrm{e}}$ siècles av. J.-C., plutôt que celle de l'esthétique de la statuaire de l'époque classique des $\mathrm{V}^{\mathrm{e}}$ et $\mathrm{IV}^{\mathrm{e}}$ siècles. De même, l'ornementation de l'armement et de l'équipement des belligérants, constituée notamment de plumes et de peaux de bêtes, évoque les guerriers tribaux africains ou amérindiens. Là encore, nous sommes loin des représentations "classiques " de l'hoplite grec présentes dans nombre de productions culturelles contemporaines. À ce sujet, il est intéressant de noter que des contradictions peuvent exister au sein de la même œuvre; sur la couverture du livre de Michel Woronoff, c'est un hoplite avec un casque de type corinthien qui apparaît au premier plan, alors que paradoxalement les illustrations à l'intérieur du livre montrent des soldats avec un armement de type mycénien. Pour en revenir à l'œuvre de Camille Le Gendre, nous noterons encore que le choix des couleurs - le rouge, ocre, noir et marron surtout -, le fourmillement des traits et leur aspect inachevé parfois font écho non seulement au titre de la bande dessinée, mais aussi à la nature épique de l'œuvre originale et aux nombreuses mêlées guerrières qui parsèment celle-ci. Tous ces éléments semblent indiquer que la démarche de l'auteur vise à retranscrire en 
termes graphiques la dimension archaïque du monde décrit dans l'Iliade. Mais il reste que cette œuvre est aux marges du deuxième groupe.

Celui-ci est composé par les adaptations, ce qui sous-entend des modifications non structurelles qui ne bouleverseraient pas le balisage référentiel habituel de l'œuvre. Les versions proposées dans ce groupe n’en comprennent pas moins des aménagements ou orientations plus conformes à l'esthétique du support, de l'auteur et finalement à "l'air du temps ». Par exemple, le livre de Michel Woronoff est une ouvre de littérature jeunesse éditée chez Casterman dans la collection Épopée ; l'éditeur en effectue la promotion en direction des collèges dans le cadre des programmes de Lettres et d'Histoire de la classe de sixième notamment. Ainsi l'une des premières pages du livre précise qu'il s'agit d'un " récit traduit et adapté du grec ancien par Michel Woronoff». Outre le fait qu'il s'adresse à un public scolaire, ce qui nécessite qu'il s'adapte au niveau d'âge et de connaissance de celui-ci, il ne retranscrit pas l'Iliade dans son intégralité, probablement pour des raisons d'accessibilité et de problématiques éditoriales. On pourrait encore citer, pour illustrer ce groupe, la recherche d'une tonalité mélodramatique (en rupture avec la dimension épique originale) dans Hélène de Troie de Robert Wise ou la transformation d'Énée en héros "herculéen " ou macistéen, sur le modèle des héros de péplum italiens, dans La guerre de Troie de Giorgio Ferroni (le héros y est d'ailleurs incarné par Steve Reeves, un acteur qui apparaissait régulièrement dans ce type de production, notamment dans le rôle d'Hercule). Dans ce dernier film, on observe en outre la relative discrétion du personnage d'Achille, au profit du Troyen Énée, indice que les auteurs, qui s'adressaient, selon la conception de l'époque, à un public populaire, auraient voulu flatter l'orgueil national en mettant en avant le personnage-clé de la légende de fondation de Rome. Dans le même sens, dans Troy de Wolfgang Petersen, outre les libertés toute hollywoodiennes prises envers l'œuvre originale, on retient l'absence des dieux, dont l'influence est déterminante dans l'Iliade. Enfin, pour ce qui est d'Ilios, de Hosegawa Hironi, c'est tout d'abord sa réalisation sous la forme d'un manga qui explique sa présence dans cette catégorie des adaptations; cependant, si l'on se penche sur la démarche poursuivie par l'auteur on constate que celui-ci semble avoir donné le rôle de protagoniste au personnage de Pâris. En effet, loin du personnage orgueilleux, sans scrupules, hédoniste et couard que représentent d'autres œuvres, celui-ci y apparaît comme un être sensible, plutôt victime de la volonté des dieux et de son destin.

Les reformulations constituent un troisième groupe, dans lequel, même si la trame générale est respectée, les libertés prises à l'égard de la source, voire des sources, originelle(s) sont bien plus marquées. Dans ce sens, Eric Shanower se propose dans Lấge de bronze de fusionner l'ensemble de la tradition littéraire sur la guerre de Troie. Sa postface est à cet égard éloquente: "L'un des éléments qui m’attirait fortement dans la guerre de Troie était l'évolution de son histoire au cours du millénaire. Tant d'écrivains, poètes, artistes et d'auteurs dramatiques, de qualité ou non, ont complété, affiné, dévoilé ou d'une façon ou d'une autre laissé leur trace sur cette histoire, jusqu’au 
moment où les transformations et les divergences semblent sans fin. Le défi de modeler toutes ces versions disparates en une seule trame cohérente m’a fasciné, et je continue à penser que c'est la partie la plus intéressante dans mon travail sur l'âge de Bronze ${ }^{4}$."

De la sorte, une autre œuvre est créée à partir d'un matériau ancien qui se retrouve émis sous une forme nouvelle. Mais, dans ce cas, le renouvellement est moindre encore que dans le quatrième groupe, celui des transpositions, ce dernier terme entendant un bouleversement de la structure traditionnelle de l'œuvre source de référence. Une raison peut en être le basculement ou l'insertion de la trame principale de celle-ci, en l'occurrence l' Iliade, dans un contexte complètement étranger au cadre originel. Il arrive aussi que certains éléments référentiels de l'œuvre source soient entremêlés avec d'autres apports, le tout dans un cadre nouveau où les éléments originels de l'Iliade ont une valeur structurante amoindrie. Ilium et Olympos de Dan Simmons ainsi que Le dernier Troyen de Valérie Mangin et Thierry Démarez, qui voient les hoplites grecs évoluer dans l'espace intersidéral, entrent dans cette catégorie.

\section{Réflexivité, rétrospectivité, désenchantement : le dépassement des bornes}

C'est aujourd'hui chose convenue d'affirmer que des productions culturelles reflètent les préoccupations, les intérêts et finalement le contexte de l'époque à laquelle elles appartiennent. Il n'en va pas autrement pour les productions culturelles qui sont étudiées ici, c'est-à-dire des films, des bandes dessinées et des romans. Il convient toutefois de signaler deux phénomènes inhérents à ces œuvres et qui semblent être plus spécifiques à notre société contemporaine : la réflexivité et la rétrospectivité, deux notions dont on constate l'influence, voire la prégnance, au sein du corpus envisagé.

Pour ce qui est de la première notion, nous nous baserons sur la définition philosophique de l'adjectif réflexif/ve : "Se dit de la conscience qui se prend elle-même pour objet ${ }^{5}$. " La réflexivité peut en conséquence être comprise comme l'action de réflexion menée sur soi-même. Le siècle précédent, et celui que nous vivons, ont à cet égard vu un formidable développement à la fois de l'individualisme et des interrogations existentialistes qui lui sont liées, ce qu'explique peut-être l'essor de la psychanalyse, des réflexions menées sur les relations entre les hommes et les femmes ou encore sur les identités masculine et féminine. Cette propension se retrouve du reste à l'échelle des groupes humains envisagés dans leur globalité, comme l'indique le développement concomitant des sciences qui ont pour objet l'étude de ces sociétés humaines dans leurs différents champs d'activités ainsi que dans l'Histoire. 
Dans cette mesure, cet aspect de la réflexivité, considérée à l'aune de l'étude des sociétés et de leurs contingences chronologiques, amène à pénétrer dans le domaine du second phénomène signalé plus haut : la rétrospectivité, terme que nous utilisons ici au lieu de "rétrospection " afin de désigner un ensemble d'attitudes ou de démarches de rétrospection. Dans ce cas également, nous partirons de la définition du mot "rétrospectif/ive " (du lat. retro, " en arrière ", et spectare, "regarder ») : "1. Qui concerne le passé et l'évolution antérieure de quelque chose ; 2. Qui se manifeste après coup, à l'évocation d'un événement ${ }^{6}$. " Dans ce sens, la rétrospectivité se conçoit comme l'attitude qui consiste à se pencher sur le passé afin de mieux le comprendre dans ses singularités et dans ses convergences avec notre époque, le tout à la lumière d'aujourd'hui, c'est-à-dire fort de la somme des démarches similaires déjà opérées au cours des époques précédentes ainsi que des avancées scientifiques et savantes contemporaines. Connaitre le passé pour lui-même donc, mais surtout le redécouvrir afin de mettre en relief les ruptures et les continuités entre les sociétés passées et les sociétés actuelles qui peuvent expliquer le contexte contemporain, une démarche qui consiste notamment à appréhender le passé pour le confronter à des problématiques du présent. Connaître le passé afin de mieux se connaître : là s'effectue la jonction entre réflexivité et rétrospectivité, là se rejoignent les interrogations de l'homme et la réflexion sur l'Homme.

Ces deux notions, réflexivité et rétrospectivité, impliquent l'exploration, voire le dépassement des limites et donc la découverte de nouveaux territoires, de nouveaux champs d'expériences, qu'ils se situent à l'échelle de l'individu ou des groupes humains. Cependant la réflexion sur l'Homme, ses aptitudes, ses travers, ses catégories psychiques, mais surtout la mise en exergue de ses outrances possibles ont progressivement renforcé, en particulier à la suite des deux conflits mondiaux, un troisième phénomène qui ne semble pas s'être tari depuis et qui correspond à ce "désenchantement du monde " dont parlait déjà Max Weber ${ }^{7}$. Ce désenchantement a, depuis, connu une évolution protéiforme. D'une part, il a conduit à une relative laïcisation des sociétés, ou tout du moins à un recul de l'emprise des religions dans le monde occidental (même si la survivance, ou plutôt la résurgence, du phénomène religieux dans nos sociétés contemporaines a été maintes fois constatée alors que toutes les prédictions consécutives au développement de ce phénomène de désenchantement incitaient à en prévoir le déclin). D'autre part, il a accompagné le déclin des idéologies eschatologiques telles que le positivisme, le progressisme ou le marxisme. De fait, nos sociétés sont en proie au sentiment d'une grande désillusion envers des idéologies perçues comme des leurres phagocytes, contraires aux aspirations et à la réalisation individuelles, des idéologies qui seraient disqualifiées par avance car présumées sectaires.

Ainsi ce désenchantement s'est manifesté par un scepticisme croissant envers les religions et le religieux, le progrès et la science, les idéologies politiques et la Politique, 
et globalement sur l'Homme et sa place à la fois dans l'histoire et dans le règne animal. Ce scepticisme lui-même a conduit à des interrogations et à une remise en question des civilisations humaines et de leurs productions, celles-ci ne bénéficiant plus, sinon du bénéfice du doute, du moins de l'argument d'autorité conféré par la caution des siècles écoulés.

En particulier, le religieux, souvent assimilé au merveilleux, est confronté le plus souvent à son désavantage au goût pour le vraisemblable et le rationnel qui caractérise l'époque contemporaine. Ce phénomène se traduit, dans les productions culturelles contemporaines, sous des formes diverses qui vont de l'absence totale des dieux - dans Hélène de Troie de Robert Wise, Làge de bronze de Eric Shanower ${ }^{8}$ et bien sûr Troy de Wolfgang Petersen ${ }^{9}$ - à leur manifestation sous une forme présentable, c'est-à-dire la

8 Cf. SHANOWER, Lâge de Bronze, p. 200 : "Chaque époque raconte la légende de la guerre de Troie de façon différente, ce qui est bien normal. L'Iliade d'Homère montre des dieux influençant directement l'action, participant même à certaines batailles. J'ai évacué les dieux de la scène, une décision pas tellement originale de ma part pour raconter à nouveau cette histoire (ce choix a été ou n'a pas été à la mode depuis des siècles), mais une décision qui je pense est pertinente dans ce monde du XXI ${ }^{e}$ siècle où beaucoup sont prompts à regarder au-delà d'eux-mêmes pour trouver des réponses ou faire porter les responsabilités. J'ai choisi de minimiser l'aspect surnaturel afin d'insister sur l'aspect humain. Les seuls éléments fantastiques que j'ai conservés sont les rêves et les visions. Et quand on y pense, ils ne sont pas si surnaturels qu'ils puissent paraître au premier abord. Tout le monde rêve. Beaucoup de gens ont des hallucinations. D'autres sont convaincus d'avoir des visions. Aux quatre coins du monde les gens pensent qu'ils communiquent avec les dieux : on appelle ça la prière. J'ai donc laissé les rêves et les visions : ils sont très humains après tout. Mais pas de dieux en chair et en os ». De même, à l'occasion d'une interview sur Internet, l'auteur déclare : "De surcroît, j'ai délibérément choisi d'effacer tous les aspects surnaturels du mythe. Et ainsi mettre l'accent sur l'aspect strictement humain du récit et rendre l'action "rationnelle". Comprendre pourquoi et dans quel but agissent les personnages. Lorsque Agamemnon sacrifie sa fille Iphigénie, il n'entend pas de voix divine lui ordonner : "Ô roi, il faut que tu...", non c'est lui, en son âme et conscience, qui doit prendre la décision fatidique. Les hommes peuvent croire aux dieux. Néanmoins, ma vision de l'épopée reste que ces derniers n'interviennent pas directement. Ils ne sont pas moteurs des actions humaines et ne guident en rien le récit».

9 " Je ne me voyais pas faire un film avec des dieux... Je ne sais pas comment j'aurais fait même avec les effets spéciaux. Il y a eu ce film dans les années 70, Le choc des Titans: c'était un kitsch ! À partir du moment où vous retirez les dieux, vous rendez les personnages responsables de leur destin. C'est plus réaliste". De fait, lors de l'épisode de la dispute entre Agamemnon et Achille, c'est Briséis, l'esclave en jeu, et non la déesse Athéna, qui met fin à ce qui aurait pu déboucher sur un affrontement armé entre les deux hommes. D'ailleurs, Agamemnon poussant un peu plus loin sa chance alors que Achille est sur le point de se retirer, déclare de façon sarcastique "Voilà le grand Achille vaincu par une esclave! ». Cependant l'ironie de ces propos peut aussi se concevoir à travers la référence à l'œuvre source, l'Iliade dans une sorte de jeu de miroir non sans une certaine auto-dérision. De même lorsque Priam se rend auprès d'Achille pour réclamer le corps d'Hector et que celui-ci lui demande " comment es-tu arrivé ici ? ", au lieu de lui répondre 
plus vraisemblable possible. Ainsi les dieux apparaissent brièvement dans La colère d'Achille de Mario Girolami, ou encore dans Hélène de Troie de John Kent Harrison, et ils participent pleinement à la trame narrative dans Le dernier Troyen de Valérie Mangin et Thierry Démarez, dans Ilios de Hosegawa Hironi ainsi que dans Ilium et Olympos. Camille Le Gendre, dans La colère d'Achille, a opté pour une autre solution encore, en retranscrivant le texte de l'Iliade, et donc l'action des dieux quand celle-ci est évoquée, mais en ne représentant pas physiquement ces derniers, si bien qu'ils sont invisibles. Une autre manifestation de ce phénomène de désenchantement au sein des productions culturelles contemporaines est aussi le transfert des critiques les plus courantes effectuées de nos jours envers les religions et les religieux sur les éléments religieux appartenant au contexte propre à l' Iliade ${ }^{10}$.

Cette dernière, et même plus généralement le récit homérique, se prêtait du reste particulièrement à subir ce désenchantement, et ce pour des raisons que l'on pourrait presque qualifier de consubstantielles. En effet, pour ne rien dire des interrogations sur l'identité de son (ou ses) auteur(s), il ne faut pas perdre de vue qu'à l'origine, l' Iliade est issue de la tradition orale. De plus, si l'on conçoit qu'elle a été composée par un même auteur, elle l'aurait été entre le $\mathrm{XI}^{\mathrm{e}}$ et le $\mathrm{IX}^{\mathrm{e}}$ siècles av J.-C., narre des événements qui se seraient déroulés vers le $\mathrm{XII}^{\mathrm{e}}$ siècle (tout en mêlant des éléments qui appartiennent à différentes époques comprises entre ces deux dates) et a été transposée vers le VIII siècle sous forme écrite, devenant de ce fait une version officielle. Elle porte ainsi en elle cette " inter-temporalité » à laquelle sont confrontés, et se confrontent, les auteurs contemporains. Cette inter-temporalité est ainsi un ensemble où s'entremêlent des références de l'Iliade, des éléments supposés appartenir au contexte grec pris au sens large, des apports liés à des erreurs ou à des emprunts, et enfin des réflexions et des points de vue contemporains. Le tout s'accentue lorsque, comme dans les romans de Dan Simmons ou la bande dessinée Le dernier Troyen, les auteurs placent le récit dans un contexte futuriste. Plusieurs choix s'offrent alors à eux : parler du passé au présent, du présent au passé ou anticiper le futur à l'aide du passé. Un tel estompage des frontières temporelles laisse augurer de nouveaux espaces, livrés au défrichement des plus folles spéculations utopiques. De fait, même dans ses concrétisations les plus raisonnables ou du moins vraisemblables, la réalisation d'une production faisant référence à une œuvre du passé est toujours l'occasion d'un questionnement voire d'une confrontation avec les nouvelles valeurs en cours dans la société de l'œuvre « seconde ». Dans cette optique, ce

comme dans l'Iliade qu'un dieu demeuré bienveillant à l'égard des Troyens lui a permis de se rendre jusqu'à sa tente, il réplique de façon assez impertinente au regard de la situation « je connais mon propre royaume mieux que les Grecs je crois ?".

Par exemple, dans plusieurs des œuvres étudiées, lors du passage où les Troyens découvrent le fameux cheval de bois laissé par les Grecs, le personnage qui insiste pour que le cheval entre dans la cité de Troie afin d'être consacré comme un don envers les dieux est un prêtre. 
sont les potentialités que permet l'Antiquité en termes de création de nouveaux Mondes, de nouvelles utopies et donc sa fonction de "matrice " qui expliqueraient la sollicitation renouvelée de cette période en vue d'évoquer des questions ou des récits qui lui sont allogènes. Ceci explique peut-être aussi pourquoi certains personnages des œuvres étudiées se comportent comme s'ils avaient une vision omnisciente de l'œuvre, ou du moins comme s'ils connaissaient non seulement l'avenir des événements auxquels ils sont confrontés dans le récit, mais en plus leur impact et leur réception auprès des générations futures. Tout se passe comme si le personnage effectuait une mise en perspective quasi historique du récit ${ }^{11}$.

De même, il ne faut pas oublier que la matière de l'Iliade est constituée de références et d'éléments légendaires et mythologiques. Or l'une des conséquences du désenchantement consiste en une sorte d'entreprise de démythification, de démystification. Une démarche qui passe à la loupe les idées reçues, les topoi, les références établies et autres passages obligés afin de voir ce qu'il en reste une fois que l'on a démonté la mécanique et les mécanismes intimes qui, à l'instar des tours de magie, procurent le charme de l'enchantement, aussi longtemps que les protège le flou artistique du mystère.

\section{Nouveaux fronts, nouvelles versions}

Parmi les manifestations conjuguées de la réflexivité, de la rétrospectivité et du désenchantement dans les versions contemporaines de la Guerre de Troie, nous nous attacherons dans un premier temps aux dialogues qui sont censés retranscrire des scènes rapportées dans le récit homérique. En effet, les personnages y utilisent parfois un langage familier, voire grossier, qui tranche avec la "matière première ", qui se voulait poétique et épique. Il peut s'agir d'introduire ainsi le langage de la quotidienneté, et donc du vraisemblable, dans le discours épique, de façon à le rendre moins ampoulé, moins grandiloquent et moins édulcoré. Le récit devient alors plus concret, plus proche, plus familier du lecteur, de son expérience et, qui sait, de ses préoccupations. Cette tendance est illustrée par le film de Robert Wise, Hélène de Troie, qui rappelle par

11 Dans Troy, Thétis, la mère d'Achille, illustre ce point de vue lorsqu'elle énonce le célèbre choix d'Achille : "Si tu restes à Larissa tu auras une vie heureuse, mais lorsque les enfants de tes enfants seront morts, on aura oublié ton nom. Si tu vas à Troie tu connaîtras la gloire. On écrira l'histoire de tes exploits pour des milliers d'années à venir et le monde n'oubliera jamais ton nom ! ». C'est finalement ce qui décide Achille à aller se battre aux côtés des Achéens De même, lorsque Hector lui demande "pourquoi es-tu venu te battre?", Achille lui répond "dans un millier d'années on parlera toujours de cette guerre " et bien qu'Hector lui rétorque "dans un millier d'années même la poussière de nos os aura disparu ", Achille persiste en disant "c'est vrai, mais nos noms ne seront pas oubliés ". Pour prendre un autre exemple, parmi bien d'autres, les propos d'Ulysse, dans Hélène de Troie (2003), révèlent aussi l’omniscience des personnages : "Ménélas a raison, ma femme a des admirateurs à sa porte, elle ne va pas tisser indéfiniment ». 
bien des aspects le mélodrame, un aspect rendu par des postures ou des tournures quelque peu outrées ainsi que par des attitudes, des situations ou des répliques qui semblent en décalage par rapport à la tonalité et l'intensité à laquelle on s'attendrait étant donné le sujet épique abordé. Cet aspect est notamment illustré par l'insistance dans le film sur la situation d'un Ménélas archétype du mari trompé : ventripotent, fat et veule, mais cependant inconsolable de la trahison de "son Hélène ", au point de perdre la fière prestance qui lui avait permis de tenir tête à Achille au début du film. La nouvelle de l'infidélité d'Hélène le décompose littéralement et, au final, il apparaît pathétique dans sa souffrance, au point que même ses comparses achéens se gaussent de son infortune conjugale. C'est aussi à travers le jeu des acteurs que se manifeste dans toute son ampleur, sinon le vaudeville, du moins le mélodrame. À la nouvelle de l'enlèvement d'Hélène, Ulysse, le premier, lance la charge à son habitude, de façon subtile et ironique : "Elle a dû se débattre, appeler au secours et hurler de terreur "; l'expression de son visage ainsi que les regards et les sourires entendus échangés entre Patrocle et Achille à la suite de ces paroles en disent long sur le crédit qui leur est accordé. Plus loin, Ulysse surenchérit sur l'emballement d'Agamemnon pour motiver les autres rois grecs à la vengeance envers Troie : "Quelle honte et une honte pour la Grèce entière ! ", dit-il avec emphase. Plus loin encore, Agamemnon continue à exploiter le filon de l'indignation, alors qu'en réalité, il projetait depuis longtemps d'attaquer Troie. Ne se sentant plus de joie après s'être assuré en un coup d'œil du soutien tacite d'Ulysse, il prend sa plus belle voix et déclare à l'ensemble des rois : "Un devoir des plus sacrés m'oblige d'être votre chef pour aller venger l'honneur de mon frère ». De même, plus loin, la brouille entre Achille et Agamemnon prend des allures de dispute d'ivrognes. Celle-ci, réduite à sa plus simple expression, a lieu au cours d'un banquet de type orgiaque ; les deux protagonistes sont tous deux avinés et Agamemnon ne paraît pas prendre au sérieux les menaces d'Achille.

Autre plan sur lequel réflexivité, rétrospectivité et désenchantement se rencontrent : le développement d'une réflexion sur les influences réciproques des œuvres entre elles, chacun s'inspirant d'une œuvre précédente (appelons-la l'œuvre source), conscient de prendre lui-même place dans une tradition qui l'a précédé, conscient aussi de pouvoir lui-même faire date et devenir à son tour un modèle. Une telle perception est une incitation perpétuelle au concours, un appel permanent à l'agôn: chaque auteur propose son interprétation, son adaptation, son originalité et au final sa version, à partir du matériau culturel qui constitue sa source de départ.

Cette problématique est omniprésente dans Ilium de Dan Simmons. Dans ce livre, l'intrigue mêle extraits et références explicites de l'Iliade, science-fiction, et finalement des réflexions très contemporaines sur un $\mathrm{XX}^{\mathrm{e}}$ siècle conçu dans l'œuvre comme un passé déjà ancien. À la multiplicité et l'entremêlement des intrigues et des protagonistes, Dan Simmons ajoute l'interaction, sinon d'époques, du moins d'espaces-temps. Résumons : sur la planète Mars, dans un temps futur (par rapport au $\mathrm{XX}^{\mathrm{e}}$ siècle), les 
dieux vivent dans leur domaine Olympos, d'où ils observent les déroulements de la guerre de Troie sur la plaine d'Ilium, et ce grâce aux récits et aux rapports effectués par d'anciens scoliastes d'époques différentes, notamment $\mathrm{du} \mathrm{XX}^{\mathrm{e}}$ siècle, comme Hockenberry, le héros principal, ressuscités par les dieux afin de les servir. La grande occupation de ces scoliastes est de vérifier que le récit d'Homère est conforme aux événements qui se déroulent sous leurs yeux dans la plaine d'Ilium, et le moindre décalage les interpelle. Mais, tout comme dans l'Iliade, les dieux interviennent parfois en personne dans le cours des événements. Ces différentes interactions provoqueront finalement une sortie de rails de la trame du récit homérique pour aboutir à la fin du premier tome à une situation où Achéens et Troyens s'allient pour combattre les dieux et assiéger leur domaine Olympos.

La question de l'intertextualité structure toute l'œuvre, et les évocations récurrentes d'auteurs tels que Shakespeare ou Proust ont certes pour effet de replacer Homère parmi ses pairs en littérature, mais aussi de remettre dans le rang, à sa place, une œuvre que beaucoup considèrent comme l'un des fondements de la civilisation occidentale ${ }^{12}$. De la sorte, Ilium pose la question de l'impact et de l'aura d'une ouvre " archi-référentielle ", voire de sa remise en cause - du moins, la remise en cause de la version classique dans laquelle celle-ci est habituellement transmise. Dans ce sens, le constat des succincts décalages entre le récit d'Homère et la réalité qui se déroule sous ses yeux sont pour le héros autant de motifs de jubilation qui lui permettent de battre en brèche l'autorité du " plus grand des poètes ${ }^{13}$ ".

L'on s'approche alors d'une attitude de démythification/démystification d'une œuvre dont la portée référentielle est telle que toucher à certains attendus de sa trame principale serait attenter au dogme, friser le sacrilège culturel envers une tradition plus qu'établie. Une tradition ancrée, si ce n'est figée, telle une statue de commandeur, imposant de sa stature son statut d'intouchable. Une telle ode au classicisme ne demanderait en vérité qu’à être interpellée, déridée, décrispée, voire déboulonnée, sinon par les esprits les plus irrévérencieux du moins par les plus audacieux.

12 Ainsi les deux intelligences artificielles envoyées pour enquêter sur Mars, Ophu d'Io et Mahmnut, ont respectivement pour idoles littéraires Proust et Shakespeare, et Homère ne fait pas partie de leur univers.

13 Ainsi au cours de l'épisode de la dispute entre Achille et Agamemnon, Hockenberry relève : "Nestor s'avance pour raconter qu'on se serrait davantage les coudes du temps de la guerre contre les centaures. Ce qui constitue une anomalie. Chez Homère, Achille ne s'est pas encore éclipsé au moment de ce discours et j'en prends note mentalement ». Plus loin, le protagoniste donne libre cours à sa réflexion sur le sujet : "Ce qui me fascine, c'est la rapidité avec laquelle les événements peuvent diverger du récit qu'en fait Homère. Les disparités que j'ai pu constater par le passé ... sont relativement mineures, et l'on peut les expliquer par la licence poétique, l'Iliade étant placée sous le signe d'une certaine unité de temps. Mais que se passera-t-il si les événements suivent vraiment un cours différent ?". 
Ce choix est sans conteste celui qu'a effectué Dan Simmons. Dès le début d'Ilium, Hockenberry donne le ton : « chante, ô Muse... Et tant que tu y es, ô Muse, chante la rage des dieux eux-mêmes, si capricieux et si puissants sur leur nouvel Olympe, et la rage de... Réflexion faite, ô Muse ne chante rien. Je te connais. J'ai été ton esclave et ton serviteur, ô Muse, ô incomparable salope. Et je n'ai aucune confiance en toi, ô Muse, vraiment aucune ». À travers ce personnage, l'auteur annonce en quelque sorte qu'il va jouer avec la référence originale, et toute l'œuvre va se structurer dans ce jeu constitué par les écarts entre l'Iliade et les apports personnels de l'auteur : une œuvre donc qui oscille entre transposition et reformulation, car non content de parsemer de traits et de réflexion contemporains le récit homérique, l'auteur en inscrit les extraits dans le canevas d'une œuvre de science-fiction qui, si elle réserve à l'Iliade une place d'honneur, n'en conserve pas moins sa propre autonomie. De fait, cette œuvre se révèle être un paradigme éloquent concernant la manifestation des phénomènes évoqués plus haut à savoir la réflexivité, la rétrospectivité et le désenchantement. Pour la première, c'est à travers le personnage principal Hockenberry qui est un archétype de l'antihéros contemporain, perclus de doutes et de complexes, qu'elle se manifeste. La rétrospectivité se marque notamment dans la confrontation des différentes temporalités et par une manipulation de la trame traditionnelle de l'Iliade. Enfin, comment ne pas voir l'influence du désenchantement dans l'affrontement entre les Achéens et les Troyens d'un côté et les dieux de l'Olympe de l'autre, qui se terminera par l'éradication de ces derniers.

En somme, cette étude a montré comment chaque auteur a exprimé sa propre vision, et peut-être devrait-on dire sa propre interprétation, de l' Iliade, chacun se livrant à des choix significatifs tant sur la forme que sur le fond : un support innovant tel que le manga, un graphisme spécifique, un changement de protagoniste, des omissions volontaires ou des rajouts surprenants, une tonalité irrévérencieuse au regard de la matière épique de l'œuvre première... Ce faisant, les auteurs, d'une part singularisent leur réalisation et d'autre part, répondent à une démarche globale qui traduit leur rapport particulier à l'œuvre originale.

Ces réalisations viennent ainsi prendre leur place dans la longue liste des reformulations de l'œuvre qui ont jalonné les siècles, au même titre, par exemple, que les illustrations de l'Iliade qui représentent Hector ou un autre héros du cycle troyen en armure du XVe siècle. Si l'on plonge la matière première, ou plutôt si on la fond, dans un autre contexte au risque de la dissoudre, c'est que celle-ci n'est peut-être plus l'objet premier, qu'elle est devenue une sorte de toile de fond, un répertoire de références prétexte à des discours qui ne sont plus les siens.

L'analyse de ces derniers, précisément, met en évidence des phénomènes spécifiques à notre société contemporaine : la réflexivité, la rétrospectivité et le désenchantement. Ces phénomènes, notamment le désenchantement, contribuent à accentuer la fonction de matrice de la guerre de Troie et structurent les œuvres contemporaines sur 
deux aspects. Le premier consiste en une mise à distance du merveilleux, et donc des dieux, mais aussi des classiques. Le second est la création d'un contexte d'idées, de représentations et d'attitudes, qui a lui-même une fonction de matrice d'œuvres qui, de fait, se révèlent plus autonomes par rapport à la tradition.

Audy RODRIGUEZ

11, promenade du canal, bâtiment $A$

91350 Grigny

rodriguezaudy@yahoo.fr 